University of Nebraska - Lincoln

DigitalCommons@University of Nebraska - Lincoln

Timothy J. Gay Publications

Research Papers in Physics and Astronomy

2013

Optically pumped spin-exchange polarized-electron source

Munir Pirbhai

J. Knepper

E. T. Litaker

D. Tupa

Timothy J. Gay

Follow this and additional works at: https://digitalcommons.unl.edu/physicsgay

Part of the Atomic, Molecular and Optical Physics Commons, and the Other Physics Commons

This Article is brought to you for free and open access by the Research Papers in Physics and Astronomy at DigitalCommons@University of Nebraska - Lincoln. It has been accepted for inclusion in Timothy J. Gay Publications by an authorized administrator of DigitalCommons@University of Nebraska - Lincoln. 


\title{
Optically pumped spin-exchange polarized-electron source
}

\author{
M. Pirbhai, ${ }^{1}$ J. Knepper, ${ }^{1}$ E. T. Litaker, ${ }^{1}$ D. Tupa, ${ }^{2}$ and T. J. Gay ${ }^{1}$ \\ ${ }^{1}$ Department of Physics and Astronomy, University of Nebraska, Lincoln, Nebraska 68588-0299, USA \\ ${ }^{2}$ Physics Division, Los Alamos National Laboratory, Los Alamos, New Mexico 87545, USA
}

(Received 18 August 2013; published 31 December 2013)

\begin{abstract}
We describe the operation of a prototype polarized-electron source. Rubidium vapor, contained in a cell, is optically pumped in the presence of a buffer gas. Unpolarized electrons from a tungsten filament are injected into the cell and extracted after undergoing spin exchange with the $\mathrm{Rb}$ atoms. We compare the performance of the source when different buffer gases are used. We measure a decrease in electron polarization as their injection energy increases, but find an unexpected regime at higher injection energies yielding increased electron polarization accompanied by a 40 -fold increase in current, suggesting the production of slow secondary electrons in the target cell. With ethylene, we have measured electron currents of $4 \mu \mathrm{A}$ simultaneously with electron polarizations of $24 \%$. This work offers the promise of a simple, benchtop, "turnkey" source of polarized electrons.
\end{abstract}

DOI: 10.1103/PhysRevA.88.060701

PACS number(s): $34.80 . \mathrm{Nz}, 07.77 . \mathrm{Ka}$

The use of polarized electrons is widespread in physics, from probing of the spin structure of nucleons and nuclei [1] to studying magnetic domain structure [2] and the spin dependence of atomic collisions [3]. The additional information provided by studies with incident polarized-electron beams comes at a price: The technological demands of polarized-electron sources are severe. The current state-ofthe-art source technology is based on photoemission from negative electron affinity (NEA) strained GaAs photocathodes [4,5]. The modern GaAs source can produce high-current $(\sim 1 \mathrm{~mA})$, high-brightness beams with $\geq 80 \%$ polarization. Also, importantly, it is optically reversible; the electron-spin direction can be flipped by reversing the helicity of the light producing photoemission from the GaAs. This enables a relatively straightforward diagnosis of systematic experimental error due to instrumental asymmetries. The biggest drawback of the GaAs source is that it is difficult to operate, particularly with regard to production of the NEA conditions that allow reasonable photocurrents to be extracted. In university labs, the learning curve for reliable graduate student operation of such sources is measured in months (or years); at accelerators, e.g., CEBAF, a dedicated scientific staff maintains and runs these sources. Several attempts to develop the GaAs source into a "blackbox" commercial technology have failed [6].

We are currently investigating the interaction of longitudinally polarized electrons, which are chiral, with chiral molecular targets. The observation of handedness-specific scattering provides information about novel molecular collision dynamics [3], as well as possible clues to the mystery of why all naturally occurring DNA has the same handedness [7]. In tabletop experiments with vapor targets, such as ours, destruction of the photocathode's NEA surface conditions by organic and other vacuum contaminants can make GaAs sources unusable or, at best, highly problematic. If a different, user-friendly source of polarized electrons, such as the one we describe here, were widely available, it could enable a broad range of experiments, including those involving electron scattering from chiral molecules.

We have demonstrated the proof of principle for an alternative method to produce spin-polarized electrons using spin exchange between unpolarized electrons and optically pumped $\mathrm{Rb}[8]$ :

$$
e(\uparrow)+\operatorname{Rb}(\downarrow) \rightarrow e(\downarrow)+\operatorname{Rb}(\uparrow) .
$$

In that experiment, a cold-cathode discharge provided unpolarized electrons. The gas sustaining the discharge (usually $\mathrm{N}_{2}$ ) also acted as the buffer gas for optical pumping. While this apparatus provided currents of $\sim 2 \mu \mathrm{A}$ with polarizations as high as $26 \%$ running with $\mathrm{N}_{2}$, it proved difficult to use, primarily because the optical pumping and electron generation processes were strongly coupled by their common elementthe buffer gas. A good compromise between the various demands on the system-maintaining a stable discharge, producing and extracting high currents of polarized electrons, reducing depolarizing wall collisions by inhibiting diffusion, quenching the $\mathrm{Rb}$ vapor, and limiting the gas load on the vacuum pumps - proved to be unattainable.

We report here an operationally robust polarized-electron source based on the spin-exchange concept. It has separate regions for unpolarized-electron production and $\mathrm{Rb}$ optical pumping. We compare its performance with different buffer gases, and find a large improvement in the beam polarization when $\mathrm{N}_{2}$ is replaced by ethylene. We have also discovered a different regime at higher electron injection energies where increased electron polarization is accompanied by a 40 -fold increase in current, suggesting the production of slow, secondary electrons in the target cell. The source presently displays currents and polarizations comparable to those of the earlier proof-of-principle experiment (and those of first-generation GaAs sources), but is much easier to operate, and holds the immediate promise of significantly improved performance with the potential for "turnkey" operation.

A diagram of the source is shown in Fig. 1. Unpolarized electrons are emitted thermionically from a tungsten filament. The filament is biased relative to an optical-pumping cell so that the electron energy upon arrival at the cell, $E_{i}$, can be selected between 0 and $110 \mathrm{eV}$. The electron beam is guided by an axial magnetic field extending the length of the apparatus with a nominal field strength of $0.02 \mathrm{~T}$. The electrons travel antiparallel to the optical-pumping laser beam and traverse the optical-pumping cell, drifting through the $\mathrm{Rb}$ vapor and buffer gas under the influence of a longitudinal electric field of 


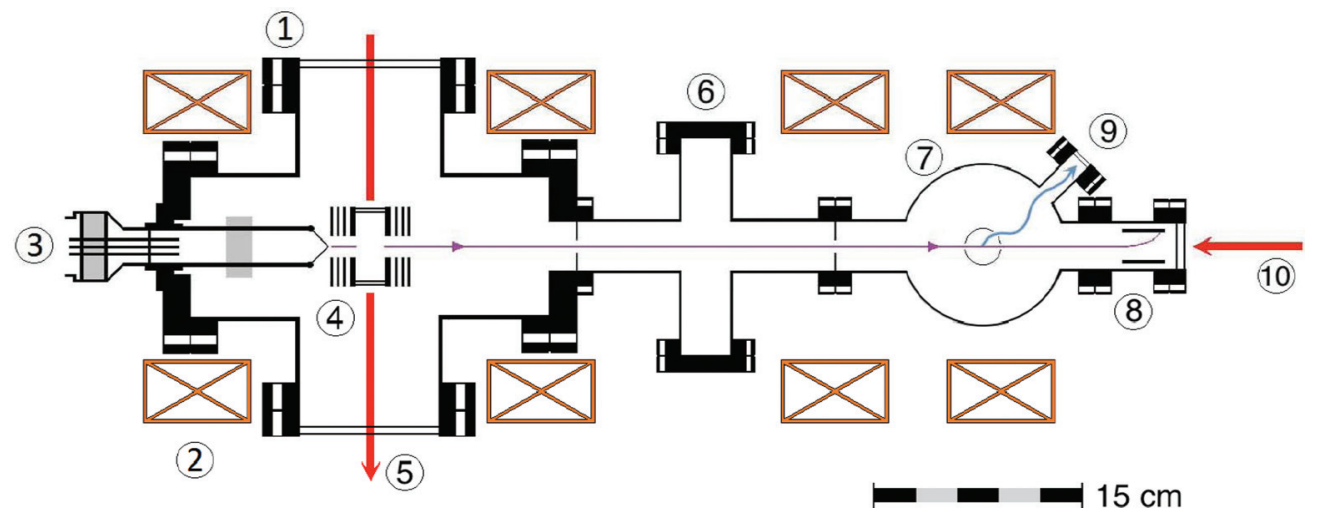

FIG. 1. (Color online) Scale diagram of the polarized-electron source showing (1) the source chamber, (2) solenoidal magnets, (3) the electron filament mounting flange, (4) the spin-transfer optical-pumping cell, (5) the transverse probe laser, (6) the differential pumping chamber, (7) the electron polarimeter, (8) the Faraday cup and pump-laser entrance window, (9) the window for the optical polarimeter used to analyze the fluorescence from a He target, and (10) the pump laser.

$\sim 150 \mathrm{~V} / \mathrm{m}$. Here they undergo spin exchange with the oriented $\mathrm{Rb}$ before being extracted through a $2-\mathrm{mm}$-diam aperture that also allows optical-pumping light into the cell. The polarized electrons then traverse a $20-\mathrm{cm}$ differential pumping chamber and enter a helium optical electron polarimeter [9].

The inside of the optical-pumping cell, made of $\mathrm{Cu}$, measures $2.8 \mathrm{~cm}$ along the source axis and is $2 \mathrm{~cm}$ in diameter. A thermally controlled reservoir feeds $\mathrm{Rb}$ vapor into the cell. Windows on either side allow a transverse probe laser to measure the $\mathrm{Rb}$ number density $N_{\mathrm{Rb}}$ using absorption spectroscopy [10]. This geometry also allows the possibility of side probing of the Rb polarization $P_{\mathrm{Rb}}$ with linearly polarized light [11]. In one experiment (Fig. 2) we were able to guide a probe laser beam that was nearly parallel to the electron beam past the thermionic filament and measure $N_{\mathrm{Rb}}$ and $P_{\mathrm{Rb}}$ with Faraday rotation $[8,12]$.

For these first experiments, we have used a somewhat inefficient optical-pumping scheme involving a single, circularly polarized pump laser, tuned to the $D 1$ transition at $795 \mathrm{~nm}$. The Sacher Cheetah laser produces up to $900 \mathrm{~mW}$ and allows frequency variation and linewidth adjustment with an

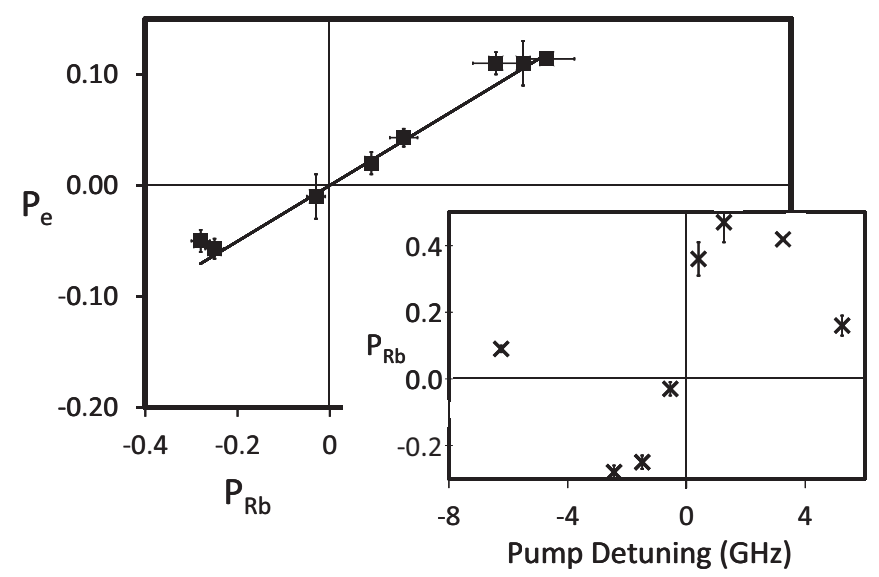

FIG. 2. The values of $P_{\mathrm{Rb}}$ and resultant $P_{e}$ are compared, displaying a linear relationship. Inset: The pump laser detuning from line center is varied in order to produce a range of $P_{\mathrm{Rb}}$ in the optical-pumping cell. externally applied voltage. Linewidth variation is achieved by superimposing a Gaussian white-noise signal on the frequency offset voltage. All measurements shown here were made with $\sim 650 \mathrm{~mW}$ of laser light with a beam diameter of $2 \mathrm{~mm}$ and a linewidth of $2 \mathrm{GHz}$ full width at half maximum (FWHM). We note that we do not employ a "repump" laser for more efficient optical pumping.

We show the linear relationship between $P_{\mathrm{Rb}}$ and the extracted electron polarization $P_{e}$ in Fig. 2. The slope of the $P_{e}$ vs $P_{\mathrm{Rb}}$ linear fit indicates the overall efficiency of the electron-Rb spin-exchange process for this combination of buffer gas species and pressure, $N_{\mathrm{Rb}}$, and $E_{i}$. For these measurements, $N_{\mathrm{Rb}}$ is $\sim 5 \times 10^{12} \mathrm{~cm}^{-3}$, the buffer gas is $\mathrm{N}_{2}$ at 200 mTorr, and $E_{i}$ is $\sim 1 \mathrm{eV}$. The range of $P_{\mathrm{Rb}}$ is achieved by pump detuning alone, and includes negative values caused by hyperfine spin reversal [13]. The inset in Fig. 2 shows how $P_{\mathrm{Rb}}$ (and hence $P_{e}$ ) varies with the pump detuning. Because the $\mathrm{Rb}$ optical-pumping process is not optimized, it is reasonable to anticipate an increase in $P_{e}$ under better conditions.

The spin-reversal phenomenon in the region of negative detuning can be used to flip $P_{e}$ independently of reversing the pump laser helicity. This provides another "handle" for the study of systematic instrumental asymmetries.

Buffer gas is introduced into the cell via a metering valve. The pressure of the gas is measured with a Convectron gauge and is known to an uncertainty of 20 mTorr. The buffer gas plays multiple important roles in the operation of the source. It reduces $\mathrm{Rb}$ depolarization by retarding diffusion to the depolarizing walls of the cell or diffusion out of the cell which necessitates replacement by unpolarized atoms. It also increases the efficiency of pump-light absorption by the $\mathrm{Rb}$ through collisional broadening. In principle, the source performance will be improved by denser $\mathrm{Rb}$ vapor, increasing the spin-exchange rate until $P_{\mathrm{Rb}}$ suffers from the effects of radiation trapping as $N_{\mathrm{Rb}}$ exceeds $\sim 10^{12} \mathrm{~cm}^{-3}[14,15]$. However, molecules with low-lying temporary negative-ion states of the appropriate energy can efficiently deexcite ("quench") Rb atoms collisionally [16]. This circumvents the radiation trapping process and allows thicker $\mathrm{Rb}$ vapors to reach high polarizations [17]. The buffer gas not only aids the optical-pumping process, it enhances the spin exchange that 


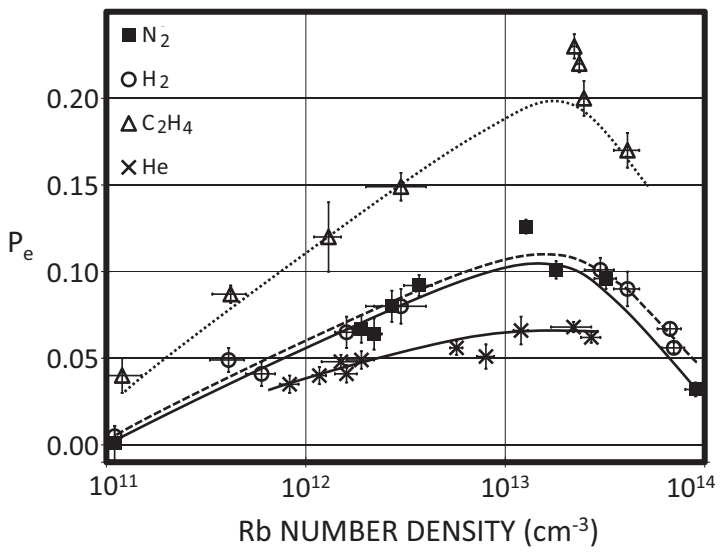

FIG. 3. Source performance with different buffer gases at 200 mTorr in the optical-pumping cell. The values of $E_{i}$ are $\sim 2$ and $\sim 4 \mathrm{eV}$ for ethylene (see text). Lines serve to guide the eye.

transfers polarization to the electrons. Collisions with a buffer gas increase the path length taken by electrons through the cell, allowing for more interactions with $\mathrm{Rb}$. These collisions also thermalize fast electrons, increasing their cross section for spin exchange. Calculations show that the total spin-exchange cross section for reaction (1) has a maximum of $\sim 10^{-13} \mathrm{~cm}^{2}$ at $30 \mathrm{meV}$. It then falls rapidly and monotonically to $\sim 3 \times$ $10^{-15} \mathrm{~cm}^{2}$ at $1 \mathrm{eV}$ [18]. Thus, we anticipate the best $P_{e}$ will occur for small $E_{i}$ when the electrons are well thermalized by the buffer gas.

Considering the many roles played by the buffer gas, one of our first experiments compares the source performance with different buffer gas species. Figure 3 displays $P_{e}$ as a function of $N_{\mathrm{Rb}}$ for a variety of buffer gases with pressures of 200 mTorr. We note that $E_{i}$ for these measurements is $2 \mathrm{eV}$, with the exception of the case of ethylene, for which $E_{i}$ is $4 \mathrm{eV}$. Ethylene is easily cracked in the vicinity of the electron gun when the electron beam is on, leaving a fine layer of conductive film on the insulators separating the gun electrodes. This makes ethylene measurements difficult with our current filament configuration, so we limited our data taking with this buffer gas to two runs. However, we include the 4-eV ethylene data because other measurements (presented below) suggest that, if anything, the performance of the source will be better at $2 \mathrm{eV}$. The ethylene data is valuable for comparison, not only because it displays the best $P_{e}$-thrice that of some other buffer gases-but also because it helps to illustrate the wide range of behaviors of the source with different buffer gases.

The data for various buffer gases yield different maxima for $P_{e}$, different values for optimal $N_{\mathrm{Rb}}$, and even different shapes for the relationship between $P_{e}$ and $N_{\mathrm{Rb}}$. This is not surprising, considering the multiple roles that the buffer gas plays in the optical-pumping cell. In fact, higher $P_{e}$ could be due to higher $P_{\mathrm{Rb}}$, more efficient spin exchange between $\mathrm{Rb}$ and electrons, or both. Of the four buffer gases, ethylene has the highest quenching cross section, $139 \times 10^{-16} \mathrm{~cm}^{2}$, while $\mathrm{N}_{2}, \mathrm{H}_{2}$, and $\mathrm{He}$ have quenching cross sections of $58 \times 10^{-16}, 6 \times 10^{-16}$, and $\ll 1 \times 10^{-16} \mathrm{~cm}^{2}$, respectively $[16,19]$. Ethylene should thus more effectively mitigate the depolarizing effects of radiation trapping, allowing for denser $\mathrm{Rb}$ vapors. Calculations indicate that ethylene causes scattered

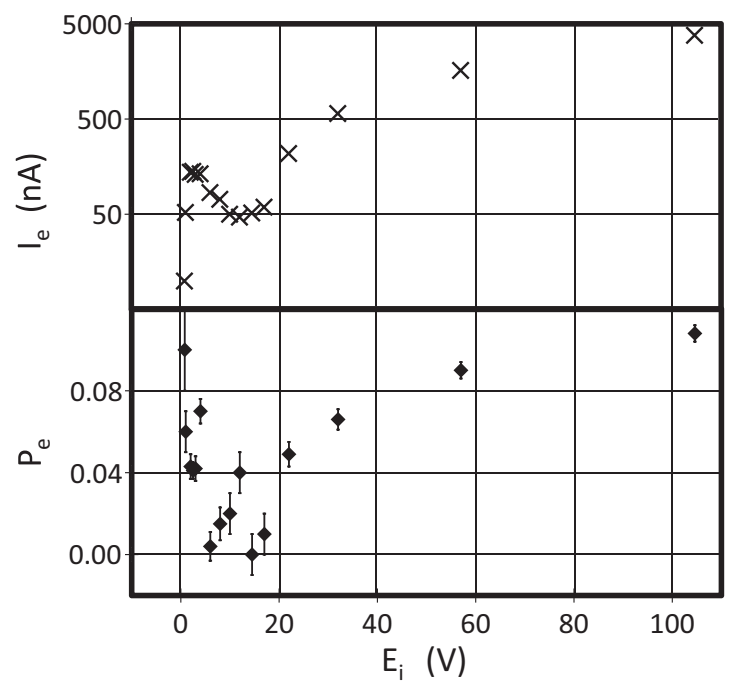

FIG. 4. Electron polarization $P_{e}$ and $I_{e}$ as a function of $E_{i}$ in the presence of 130 mTorr of nitrogen buffer gas with $N_{\mathrm{Rb}} \approx 10^{13} \mathrm{~cm}^{-3}$ and a pump wavelength and spectral width of $794.976 \mathrm{~nm}$ and $2 \mathrm{GHz}$, respectively. For $E_{i}$ below $\sim 15 \mathrm{eV}, I_{e}$ and $P_{e}$ behave in a way consistent with the energy dependence of the electron thermalization and $\mathrm{Rb}$ spin-exchange cross sections.

electrons to quasielastically thermalize 100 times faster than in nitrogen, giving them a correspondingly higher cross section for spin exchange $[18,20,21]$.

Future experiments in a source modified to allow easy implementation of a longitudinal probe laser geometry will allow separate measurements of $P_{\mathrm{Rb}}$ and $P_{e}$ such as those shown in Fig. 2. This will allow us to disentangle more thoroughly the various roles played by the buffer gas. This could, in turn, guide the choice of the best buffer gas for this application and indicate its optimal pressure. Indeed, our source makes a good laboratory for studying these phenomena.

The polarization of electrons $P_{e}$ and the total electron current $I_{e}$ from the source using a $\mathrm{N}_{2}$ buffer is shown as a function of $E_{i}$ in Fig. 4. For energies below $\sim 15 \mathrm{eV}$, the behavior of $P_{e}$ can be understood qualitatively in terms of the cross sections for electron thermalization (energy loss) and spin exchange with the $\mathrm{Rb}$ atoms [18,22]. Below this energy, $I_{e}$ is determined largely by the electron-optical properties of the system comprising the thermionic gun, $\mathrm{Rb}$ vapor cell, and extraction lenses, as determined by SIMION [23] analysis. In the same energy range, $P_{e}$ generally decreases, following the spin-exchange cross section's energy dependence. We note, however, the two local maxima for $P_{e}$ in the vicinities of 3 and $11 \mathrm{eV}$ due to energy loss of the primary electron beam and the corresponding enhancement of spin-transfer probability. These features are reminiscent of the trapped electron energy spectra of Ref. [22], associated with the production of temporary negative-ion states followed by decay to vibrationally excited levels of the $X^{1} \Sigma_{g}^{+}$ground state of $\mathrm{N}_{2}(3 \mathrm{eV})$, or inelastic excitation of the $C^{3} \Pi_{u}$ (and, possibly, the $a^{1} \Pi_{g}$ ) electronic states $(11 \mathrm{eV})$.

Above $20 \mathrm{eV}$, the source behaves quite differently, with $I_{e}$ beginning to increase rapidly. We see the largest electron current at the maximum potential difference afforded by our 
present power supply, $110 \mathrm{~V}$, where $I_{e}$ is $\sim 40$ times greater than $I_{e}$ below $17 \mathrm{eV}$. Surprisingly, this increase is accompanied by a corresponding increase in $P_{e}$. Following its initial drop, the polarization recovers completely and matches its value for the lowest $E_{i}$. This behavior seems puzzling in light of the energy dependence of reaction (1).

The large values of $P_{e}$ for $E_{i}>25 \mathrm{eV}$ lead us to believe that there are many thermal electrons in the source in this regime. We speculate that numerous secondary electrons are produced from the ionization of the $\mathrm{N}_{2}$ buffer gas by the primary electrons. These slower electrons would have a relatively high probability of undergoing spin-exchange collisions with oriented $\mathrm{Rb}$ atoms. If $N_{\mathrm{N}_{2}}$ is the number density of $\mathrm{N}_{2}$ molecules, $\sigma_{\text {ion }}$ the electron-impact single ionization cross section, and $L$ the spin-exchange cell length, we expect an appreciable number of secondary electrons produced by impact ionization whenever $\left(N_{\mathrm{N}_{2}} \sigma_{\text {ion }} L\right)$ exceeds the order of unity. Between 17 and $27 \mathrm{eV}$, the ionization cross section for $\mathrm{N}_{2}$ increases an order of magnitude to $\sigma_{\text {ion }}=$ $1.1 \times 10^{-16} \mathrm{~cm}^{2}$ [24]. For our cell length of $2.8 \mathrm{~cm}$ and a buffer gas pressure of 130 mTorr, $\left(N_{\mathrm{N}_{2}} \sigma_{\text {ion }} L\right)=1.2$ at $27 \mathrm{eV}$. Above $27 \mathrm{eV}$, the ionization cross section increases modestly to reach its maximum value at $100 \mathrm{eV}$, where $\left(N_{\mathrm{N}_{2}} \sigma_{\text {ion }} L\right)$ is 3. Alternatively, it is possible that polarized electrons are stripped directly from the $\mathrm{Rb}$ atoms and that their polarization survives. In any case, these slow electrons are guided through the spin-exchange cell by the longitudinal magnetic and electric fields applied along the beamline. We also expect that they will have an increased transmission probability because "space-charge blowup" of the beam is reduced by residual $\mathrm{N}_{2}{ }^{+}$ ions. The transport through the spin-exchange cell will be less efficient for the fast scattered primary electrons responsible for this ionization.

The process multiplying our beam current and producing large $P_{e}$ at high incident electron energy has a big impact on the performance of our source. Thus, it is important to understand its cause. To obtain evidence to confirm or refute our conjecture regarding secondary-electron production, we perform a retarding-potential analysis of the electron beam from the source when $E_{i} \approx 107 \mathrm{eV}$, and compare the results for 110 mTorr of $\mathrm{N}_{2}$ buffer gas with those for no buffer gas. The retarding potential is provided by the inner cylindrical electrode of our electron polarimeter. Smooth interpolated curves fit to these data are shown in Fig. 5. If no $\mathrm{N}_{2}$ is present, the beam comprises almost entirely electrons at $\sim 107 \mathrm{eV}$ with

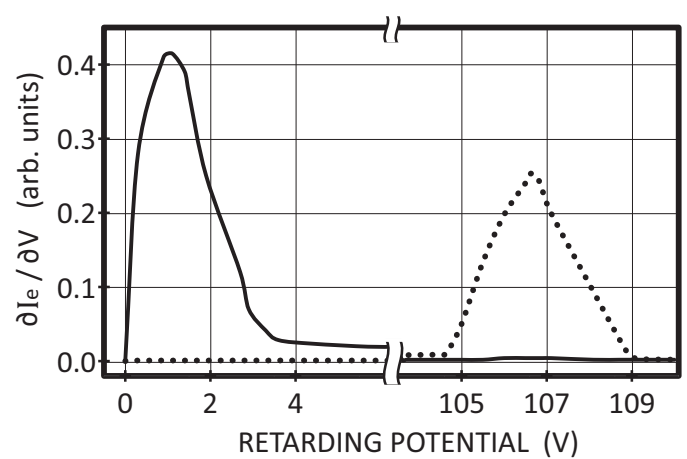

FIG. 5. Retarding field analysis of a beam with $E_{i} \approx 107 \mathrm{eV}$ passing through the optical-pumping cell. Smooth interpolated curves are fit to the values of the electron current, normalized to the current in the absence of a retarding potential. The derivatives of the interpolated curves for the cases of no buffer gas (dotted line) and 110 mTorr $\mathrm{N}_{2}$ (solid line) are shown. Electron energy widths deduced from these curves are accurate to $0.5 \mathrm{eV}$.

a FWHM energy spread of $\leq 2 \mathrm{eV}$. When $\mathrm{N}_{2}$ is introduced to the cell, the beam is dominated by slow electrons. This supports our hypothesis that secondary electrons are produced in the cell. If secondary-electron formation is indeed the source of this beneficial process, yet another factor is added to the list of requirements for an optimal buffer gas: high electronimpact ionization cross section. We note, in this regard, that the maximum total (single) electron-impact ionization cross sections for the buffer gases we studied all occur for incident electron energies in the vicinity of $100 \mathrm{eV}$, and have values of $5.5 \times 10^{-16}[25], 3 \times 10^{-16}, 1 \times 10^{-16}$, and $0.5 \times 10^{-16} \mathrm{~cm}^{2}$ [26], for ethylene, nitrogen, hydrogen, and $\mathrm{He}$, respectively.

This source is already significantly more user friendly than typical GaAs sources. We expect that, as our understanding of the detailed operational physics improves and modest mechanical improvements are implemented, true turnkey operation is possible.

We wish to thank L. Marquart, H. Batelaan, and especially P. D. Burrow for numerous helpful conversations. G. Richards and D. Ryan were involved in this effort in its early stages. This work has been supported by the NSF [Grants No. PHY0855629, No. PHY-1206067, and No. PHY-0821385 (MRI)] and has been approved for unlimited release under LANL Release No. LA-UR-13-26405.
[1] See, e.g., in Proceedings of the SPIN 2010 Conference, edited by H. Ströher and F. Rathmann [J. Phys.: Conf. Ser. 295, 011001 (2011) and references therein].

[2] See, e.g., B. Santos, S. Gallego, A. Mascaraque, K. F. McCarty, A. Quesada, A. T. N'Diaye, A. K. Schmid, and J. de la Figuera, Phys. Rev. B 85, 134409 (2012) and references therein.

[3] T. J. Gay, Adv. At. Mol. Phys. 57, 157 (2009).

[4] D. T. Pierce, in Atomic, Molecular, and Optical Physics: Charged Particles, edited by F. B. Dunning and R. G. Hulet, Vol. 29A (Academic, San Diego, 1995).
[5] T. Maruyama, E. L. Garwin, R. Prepost, G. H. Zapalac, J. S. Smith, and J. D. Walker, Phys. Rev. Lett. 66, 2376 (1991).

[6] D. T. Pierce (private communication).

[7] W. A. Bonner, Chirality 12, 114 (2000).

[8] H. Batelaan, A. S. Green, B. A. Hitt, and T. J. Gay, Phys. Rev. Lett. 82, 4216 (1999).

[9] M. Pirbhai, D. M. Ryan, G. Richards, and T. J. Gay, Rev. Sci. Instrum. 84, 053113 (2013).

[10] P. Siddons, Ph.D. thesis, University of Durham, 2011.

[11] J. M. Dreiling, E. B. Norrgard, D. Tupa, and T. J. Gay, Phys. Rev. A 86, 053416 (2012). 
[12] Z. Wu, M. Kitano, W. Happer, M. Hou, and J. Daniels, Appl. Opt. 25, 4483 (1986).

[13] E. B. Norrgard, D. Tupa, J. M. Dreiling, and T. J. Gay, Phys. Rev. A 82, 033408 (2010).

[14] D. Tupa, L. W. Anderson, D. L. Huber, and J. E. Lawler, Phys. Rev. A 33, 1045 (1986); D. Tupa and L. W. Anderson, ibid. 36, 2142 (1987).

[15] M. A. Rosenberry, J. P. Reyes, D. Tupa, and T. J. Gay, Phys. Rev. A 75, 023401 (2007).

[16] R. G. W. Norrish and W. MacF. Smith, Proc. R. Soc. London, Ser. A 176, 295 (1940).

[17] W. Happer, Rev. Mod. Phys. 44, 169 (1972).

[18] C. Bahrim, U. Thumm, and I. I. Fabrikant, Phys. Rev. A 63, 042710 (2001).
[19] E. S. Hrycyshyn and L. Krause, Can. J. Phys. 48, 2761 (1970).

[20] J. M. Warman and M. C. Sauer, J. Chem. Phys. 62, 1971 (1975).

[21] L. G. Christophorou, in Electron-Molecule Interactions and Their Applications, Vol. 2 (Academic, Orlando, 1984), p. 166.

[22] G. J. Schultz, Phys. Rev. 125, 229 (1962).

[23] SIMION 8.0, http://www.simion.com

[24] Y. Itikawa, J. Phys. Chem. Ref. Data 35, 31 (2006).

[25] S. Popović, S. Williams, and L. Vušković, Phys. Rev. A 73, 022711 (2006).

[26] D. Rapp and P. Englander-Golden, J. Chem. Phys. 43, 1464 (1965) 